Diversity of Research in Health Journal / Revue de la Diversité de la Recherche en Santé

Vol 4, January / Janvier 2021 - ISSN: 2561 -1666 DOI : 10.28984/drhj.v4i1.327

\title{
L'analyse de livres gradués (Escalire) par entremise du système d'analyse de texte par ordinateur (SATO)
}

\section{A. Godin, M. Minor-Corriveau \& R. Bélanger}

DRHJ/RDRS 2021, 4, pp.156-171

\author{
Alexie Godin \\ Étudiante à la maîtrise, agodin@laurentian.ca \\ École d'orthophonie, Faculté de la santé \\ Université Laurentienne, Sudbury (ON), Canada
}

Michèle Minor-Corriveau, M.Sc.S. Ph.D

Professeure agrégée, Orthophoniste, mminorcorriveau@laurentian.ca

École d'orthophonie, Faculté de la santé

Université Laurentienne, Sudbury (ON), Canada

Roxanne Bélanger, M.Sc.S. Ph.D.

Professeure agrégée, Orthophoniste, rbelanger@1aurentian.ca

École d'orthophonie, Faculté de la santé

Université Laurentienne, Sudbury (ON), Canada

\section{Résumé}

Les livres gradués permettent aux élèves au cycle primaire de lire des textes qui correspondent au niveau attendu selon leur niveau scolaire et leurs compétences. Les livres d'une série de livres gradués, comme ceux d'Escalire sont déjà classés selon leur niveau de complexité lié aux niveaux scolaires de façon étalonnée (Éditions Passe-Temps, 2014). Cette série contient 156 livres au total, qui sont répartis en 15 niveaux, de la $1^{\text {re }}$ à la $4^{\mathrm{e}}$ année, soit de A à O. Cette échelle correspond aux niveaux établis par Fountas et Pinnell (2012). Cette série de livres gradués consiste en un programme d'intervention en lecture ciblant les élèves de la première à la quatrième année du cycle primaire. De tels programmes d'intervention en lecture peuvent mener l'élève vers l'acquisition et l'amélioration de la lecture en fonction de ses habiletés et son niveau scolaire. L'objectif de cette étude était de déterminer s'il y a corrélation entre les niveaux de lecture attribués aux livres de la série Escalire et le niveau de complexité indiqué par le système d'analyse de texte par ordinateur (SATO), pour ces mêmes livres. Les résultats ont démontré une corrélation modérée $(\mathrm{r}=0.668, p<0,001)$ et un pourcentage d'accord de 35,26\%. Somme toute, $95,51 \%$ des livres de la série Escalire se sont trouvés soit au niveau correspondant à l'échelle SATO ou à un niveau près (soit un niveau supérieur ou inférieur à ce qui a été proposé par SATO). À partir d'études comme celle-ci, les enseignants seront en mesure de différencier leur pédagogie en ciblant des livres qui correspondent aux habiletés de leurs élèves.

Mots Clés : Escalire, livres gradués, échelle de lecture Fountas et Pinnell, niveau de complexité, SATO

\begin{abstract}
Leveled readers are books that allow students in the primary cycle to read texts that correspond to the expected level according to their grade level and skills. A series of leveled readers, such as those by Escalire, are already calibrated according to their level of complexity related to grade levels (Éditions Passe-Temps, 2014). This series contains 156 books in total, which are divided into 15 levels, from A to O (grade 1 to grade 4). This scale corresponds to the levels established by Fountas and Pinnell (2012). This series of leveled readers is a reading intervention program targeting elementary students from grades 1 to 4 . Reading recovery programs such as this can lead students to acquire and improve reading abilities according to their skill and grade level. The objective of this study was to determine the correlation between the reading levels attributed to the books in the Escalire series and, the level of complexity of these books as indicated by SATO, a text-based computer analysis system. The results indicated a significant correlation of medium size $(\mathrm{r}=0.668)$ and a percentage of agreement of $35.26 \%$. In total, $95.51 \%$ of the books in the Escalire series were either at the level corresponding to the SATO scale or within one level (either one
\end{abstract}


Diversity of Research in Health Journal / Revue de la Diversité de la Recherche en Santé

Vol 4, January / Janvier 2021 - ISSN: 2561 -1666 DOI : 10.28984/drhj.v4i1.327

level higher or lower than what had been proposed by SATO). Studies such as these allow teachers to differentiate their instruction by choosing books that correspond to their student's reading level and skill.

Keywords : Escalire, leveled readers, Fountas and Pinnell, level of complexity, SATO

\section{Introduction}

Des séries de livres gradués sont créées pour permettre aux élèves d'accéder à des livres correspondant à leurs habiletés en lecture (Éditions Passe-Temps, 2014). La série Escalire en est un exemple parmi d'autres. Escalire est un programme d'intervention en lecture qui a été créé au Québec par les Éditions Passe-Temps (2014). Le but principal de ce programme est de fournir aux élèves de la première année à la quatrième année des livres qui correspondent à leurs habiletés en lecture. Ce programme comprend de 156 livres gradués, allant de livres simples, à des livres plus complexes en lecture. Les livres sont répartis en 15 niveaux - soit de $\mathrm{A}$ à $\mathrm{O}$ - qui suivent les niveaux tels qu'établis par Fountas et Pinnell (2012). Chaque livre contient alors des critères précis qui correspondent aux niveaux auxquels ils sont attribués ainsi que des statistiques relatives aux mots et à la complexité du texte. Les niveaux de $\mathrm{A}$ à $\mathrm{D}$ sont les niveaux de base et contiennent 60 livres (niveaux $\mathrm{A}$ et $\mathrm{B}=18$ livres chacun; niveaux $\mathrm{B}$ et $\mathrm{C}=12$ livres chacun), les niveaux de $\mathrm{E}$ à $\mathrm{H}$ sont les niveaux intermédiaires qui contiennent au total 48 livres (niveaux E, F, G et $\mathrm{H}=$ 12 livres chacun) et les niveaux de $\mathrm{I}$ à $\mathrm{O}$ sont les niveaux supérieurs qui contiennent, somme toute, 48 livres (niveau $\mathrm{I}=12$ livres; niveaux $\mathrm{J}, \mathrm{K}, \mathrm{L}, \mathrm{M}, \mathrm{N}$ et $\mathrm{O}=6$ livres chacun). Plus les livres sont complexes, moins il y en a par niveau. Ceci permet donc à l'élève d'obtenir plusieurs occasions d'exposition à des livres de complexité équivalente à chaque niveau. Plus on progresse dans la série, plus les livres deviennent complexes : le texte augmente en longueur, il y a moins d'images, la syntaxe est plus complexe et le lexique, plus varié et moins fréquent. L'ensemble des livres de la collection Escalire exploite les mêmes personnages, ce qui peut permettre de stimuler la motivation des jeunes lecteurs ainsi que de créer un attachement aux personnages tout au long de sa progression dans le programme. Tous les livres respectent des critères particuliers quant aux éléments suivants : la mise en page, la longueur, le lexique, la syntaxe, le type de texte et de contenu et la relation texte-image (Les Éditions Passe-Temps, 2014).

L'utilisation des livres gradués peut s'avérer très avantageuse. Ces livres qui ont été créés précisément pour correspondre aux critères d'un niveau particulier peuvent faciliter l'acquisition de la lecture pour les élèves. En effet, plusieurs études appuient l'emploi de livres gradués (Cunningham, Spadorcia, Erickson, Koppenhaver, Strum et Yodler, 2005 ; Fountas et Pinnell, 1999 ; Hastings, 2016 ; Tiernan, 2016) et font en sorte qu'ils permettent aux élèves de reconnaître plus facilement les mots fréquents, c'est-à-dire des mots qui se retrouvent dans les textes et qui sont pratiqués afin que les élèves en viennent à les reconnaître de façon automatique (Leonard, 2012). Seals (2013), pour sa part, confirme que les livres gradués améliorent la fluidité orale en lecture ainsi que le niveau de compréhension des élèves. Afin d'améliorer ces composantes essentielles chez soi, il est important que les élèves suivent un programme de lecture, comme le 
Diversity of Research in Health Journal / Revue de la Diversité de la Recherche en Santé

Vol 4, January / Janvier 2021 - ISSN: 2561 -1666 DOI : 10.28984/drhj.v4i1.327

programme Escalire, ce qui leur permet de lire des livres qui se situent dans leur zone proximale de développement (Vygotsky, 1978), soit à leur niveau de lecture.

Dans la série de livres Escalire (2014), les livres qui correspondent aux niveaux de base (A à $\mathrm{D})$ contiennent moins de pages et de mots et contiennent des groupes de mots répétitifs, une structure de phrase simple, des thèmes familiers ainsi que plusieurs images isolées qui sont assez étroitement reliés au texte. Les niveaux correspondant aux niveaux intermédiaires ( $\mathrm{E}$ à $\mathrm{H}$ ) contiennent un nombre de pages plus élevé, des mots fréquents ainsi que des mots plus complexes (p. ex., des adverbes plus complexes comme absolument, quelquefois et tranquillement) (Ministère de l'Éducation, du Loisir et du Sport, 2014), des structures de phrases plus variées, des thèmes familiers et imaginaires, en plus d'images séquentielles qui sont retrouvées à la fin des livres pour permettre à l'élève de raconter l'histoire en ses propres mots. Les livres classés dans les niveaux supérieurs ( $\mathrm{I}$ à $\mathrm{O}$ ) sont munis d'un nombre de pages encore plus élevé, une augmentation du nombre de phrases par page, des mots nouveaux, des structures de phases plus complexes et d'autant plus variées, des thèmes familiers et imaginaires, ainsi que des images séquentielles.

Le système d'analyse d'un texte par ordinateur (SATO) est un outil qui permet de déterminer le niveau de lecture d'un livre en fonction de critères divers. On retrouve SATO sur le site www.ling.uqam.ca/sato, créé au Québec par Daoust, Laroche et Ouellet (1996). Dans le logiciel SATO la catégorie SATO-CALIBRAGE est utilisée afin d'indiquer le niveau de difficulté d'un texte en utilisant des critères par rapport à la syntaxe et au vocabulaire. Aussi cette souscatégorie permet-elle d'établir si un texte respecte le niveau ciblé tout au long de sa rédaction, ou pas. Afin d'assigner un niveau de complexité à un texte, SATO-CALIBRAGE emploie diverses variables, telles que : le pourcentage de phrases de plus de 30 mots, le pourcentage de points à la fin de la phrase, le pourcentage de mots non familiers ou inconnus, le pourcentage de forme fonctionnelle difficile accompagné du pronom «vous », le pourcentage de fréquence du pronom personnel «tu», le pourcentage de nombre de phrases par livre, le pourcentage de points d'exclamation, le pourcentage de pronoms relatifs, le pourcentage de mots contenant 9 lettres ou plus, le pourcentage de la préposition « en », le pourcentage du déterminant «l' », le pourcentage de verbes conjugués, le pourcentage d'adjectifs et le pourcentage de phrases contenant plusieurs mots non familiers. La présence ou l'absence de certaines variables est comptabilisée et entraîne un indice de valeur. SATO-CALIBRAGE utilise l'indice Gunning Fog afin d'exécuter un calcul selon le pourcentage obtenu pour chacune des variables mesurées. Ce résultat permet d'identifier le niveau scolaire durant lequel il serait attendu que l'élève puisse faire la lecture du texte aisément. Ce site rend accessible une droite de régression basée sur le niveau de complexité du texte en fonction de l'année scolaire (soit de la première année jusqu'à la onzième année). Dans cette étude, SATO est utilisé pour analyser les livres de la série Escalire. Lorsque ces textes sont saisis dans le logiciel, un indice de valeur a été attribué aux livres, en fonction des variables indiquées ci-dessus. Pour dire plus simplement, SATO établit le niveau de complexité des livres. L'indice de valeur correspond à peu près aux classes d'enseignement ( $p$. ex. le niveau 1.3 correspondrait à la $1^{\text {re }}$ année, alors que le niveau 4.6 correspondrait à la $3^{\mathrm{e}}$ année) (Daoust et coll., 1996). 
Diversity of Research in Health Journal / Revue de la Diversité de la Recherche en Santé Vol 4, January / Janvier 2021 - ISSN: 2561 -1666 DOI : 10.28984/drhj.v4i1.327

Tableau 1 : L'indice de valeur correspondant aux classes d'enseignement

\begin{tabular}{ll}
\hline Les classes d'enseignement & Valeurs de l'indice \\
\hline 1 re & $0-2.8$ \\
$2 \mathrm{e}$ & $2.9-3.8$ \\
$3 \mathrm{e}$ & $3.9-4.9$ \\
$4 \mathrm{e}$ & $5-5.5$ \\
$5 \mathrm{e}$ & $5.6-6.4$ \\
$6 \mathrm{e}$ & $6.5-6.7$ \\
$7 \mathrm{e}$ & $6.8-7$ \\
$8 \mathrm{e}$ & $7.1-8.3$ \\
$9^{\mathrm{e}}$ & $8.4-8.8$ \\
$10^{\mathrm{e}}$ & $8.9-9.1$
\end{tabular}


Diversity of Research in Health Journal / Revue de la Diversité de la Recherche en Santé Vol 4, January / Janvier 2021 - ISSN: 2561 -1666 DOI : 10.28984/drhj.v4i1.327

Les textes qui correspondent au niveau attendu pour un niveau scolaire particulier donnent l'occasion aux élèves de bien développer et de consolider leurs habiletés en lecture (Ministère de l'Éducation de l'Ontario, 2003). Galloway-Bell (2003) souligne que la lecture à livres nivelés augmente la confiance, l'estime de soi et la réussite scolaire des élèves. Le programme-cadre du curriculum Français (Ministère de l'Éducation de l'Ontario, 2006) vise à faire pratiquer la lecture régulièrement afin de développer la motivation, la fluidité ainsi qu'une bonne compréhension du texte. De plus, les élèves doivent lire des livres de complexité croissante afin d'améliorer leurs habiletés de manière progressive. Dans l'éventualité où l'élève est confronté à des livres qui correspondent à un niveau de lecture inférieur à ses capacités de lecture, l'élève risque alors d'être sous-stimulé au niveau de la lecture. Ce manque de défi à relever pourra nuire à ses habiletés. Inversement, si l'élève est confronté à des livres qui correspondent à un niveau de lecture qui excède ses habiletés, alors cela pourrait mener à un traitement inefficace du texte et l'élève est alors à risque d'être peu motivé à lire (Guthrie et Davis, 2010). Il est donc essentiel de présenter à l'élève des textes en lien avec ses habiletés en lecture en lui présentant des défis qui correspondent à ce qui est attendu en fonction de sa zone proximale de développement (Vygotsky, 1978). L'avantage de mettre au service des élèves une série de livres gradués comme Escalire, c'est qu'ils sont déjà classés selon un niveau de complexité étalonné qui respecte des critères de manière équilibrée. Cependant, les niveaux scolaires auxquels sont attribués ces livres n'ont pas été étalonnés ni validés en Ontario, soit une province où le français est une langue minoritaire. Une étude suggère que le système employé pour juger de la complexité d'un livre manque de fiabilité et qu'il y a une variation inter- et intra niveaux de la qualité de ces livres (Pitcher et Fang, 2007). Il est donc important de savoir si les livres sont classés au niveau scolaire qui correspond aux habiletés des élèves en Ontario. Avant de procéder à une validation des livres auprès des élèves en Ontario français, il importe de vérifier si les livres de la série Escalire, qui a été étalonnée en fonction des niveaux établis par Fountas et Pinnell (2012), soit de A à O, respectent la progression proposée par SATO. L'étude cherche alors à répondre aux questions de recherche suivantes :

1. Quelle est la corrélation entre (a) les niveaux de lecture attribués aux livres de la série Escalire en fonction de Fountas et Pinnell (2012) et (b) le niveau de complexité tel qu'indiqué par le système d'analyse de texte par ordinateur (SATO) (Daoust et coll., 1996)?

2. Quel est le pourcentage d'accord entre (a) les niveaux scolaires ciblés par les livres de la série Escalire en fonction de Fountas et Pinnell (2012) et (b) l'indice du système d'analyse de texte par ordinateur (SATO) (Daoust et coll., 1996) ?

En tant qu'hypothèse pour la première question, les chercheuses supposent que les niveaux attribués par SATO et Escalire ${ }^{1}$ devraient être assez bien corrélés. SATO et Escalire devraient classer les livres plus simples au bas de leurs échelles respectives et les livres plus complexes devraient être classés à un niveau plus élevé de leurs échelles respectives. Les niveaux scolaires

\footnotetext{
${ }^{1}$ Le classement des livres par niveau scolaire en fonction de la clientèle ciblée (français langue maternelle, immersion française, Échelle de Fountas et Pinnell, Échelle de Clay) de diverses collections publiées par Chenelière peut être repéré à https://www.cheneliere.ca/FTP/livre numerique/Tableau_de_correlation.pdf (Chenelière, 2014).
} 
Diversity of Research in Health Journal / Revue de la Diversité de la Recherche en Santé

Vol 4, January / Janvier 2021 - ISSN: 2561 -1666ＤOI : 10.28984/drhj.v4i1.327

tels qu'identifiés par Chenelière (2014) correspondent au même groupe d'âge que les élèves soient en Ontario ou au Québec (un élève en $1^{\text {re }}$ année doit avoir 6 ans avant le 31 décembre de l'année en cours). Aucune étude n'a tenté de vérifier si les niveaux attribués par Fountas et Pinnell (2012) correspondent à l'échelle employée par SATO.

\section{Méthode}

\section{Matériel}

Le système d'analyse d'un texte par ordinateur (SATO) est un outil qui permet de déterminer le niveau de lecture d'un livre en fonction de critères précis. Afin d'évaluer la validité de ce logiciel, 679 textes ont été distribués aux écoles primaires et secondaires dans la région du Québec. L'évaluation de ces textes a été faite à l'aide d'une grille d'évaluation du Ministère de l'Éducation, du Loisir et du Sport (MELS) (2006). Cette étude a permis aux chercheuses d'identifier 14 variables à retenir lorsqu'un système d'ordinateur cherche à attribuer un niveau de complexité à un texte. La présence ou l'absence de certaines variables donne un indice de valeur au livre, soit un niveau de complexité donné qui détermine le niveau scolaire idéal pour la compréhension de ceux-ci. Dans cette étude, les chercheuses vont saisir les textes de la série de livres gradués Escalire dans ce logiciel afin de déterminer leurs niveaux scolaires ciblés par SATO.

La série de livres gradués Escalire contient 156 livres. Ils sont divisés en 15 niveaux en utilisant l'échelle de Fountas et Pinnell (de A à O). D'après le site d'Escalire (Éditions PasseTemps, 2014), les élèves devraient être rendus au niveau $\mathrm{F}$ à la fin de la première année, au niveau $\mathrm{J}$ à la fin de la deuxième année, au niveau $\mathrm{M}$ à la fin de la troisième année et au niveau $\mathrm{O}$ à la fin de la quatrième année (2014). Dans cette étude, cette série de livres sera utilisée. Les chercheuses vont comparer les niveaux scolaires qui ont été établis par Escalire, à ceux déterminés par l'indice de valeur attribué par SATO-CALIBRAGE.

\section{Procédure}

\section{Collecte des données}

Afin de faire cette analyse, tous les textes des livres de la série Escalire ont été saisis dans un logiciel de traitement de texte et insérés dans le logiciel SATO-CALIBRAGE. Une fois les textes insérés, certains mots n'ont pas été reconnus par le logiciel, alors il fallait sélectionner la nature de ces mots (par exemple, pour le mot « ferme », il fallait alors préciser s'il prenait le rôle d'un nom commun ou celui d'un verbe). Une fois cette étape franchie, les chercheuses ont éliminé certains mots de l'analyse. Des onomatopées (par exemple, «wouf ») et des interjections (par exemple, «hourra ») ont été éliminées puisqu'elles étaient traitées comme étant des mots non familiers ou inconnus, ce qui avait pour résultat d'augmenter le niveau de complexité des livres attribués par SATO-CALIBRAGE. Par contre, ces types de mots se trouvent dans des livres appartenant aux niveaux simples. Après avoir effectué ces étapes et afin d'en aboutir à la validation des textes, SATO-CALIBRAGE fournit deux tableaux indiquant le classement du texte. Le premier tableau considère la longueur des textes tandis que le deuxième tableau ne considère pas la longueur du texte. Selon les chercheurs, Daoust et coll. (1996), le deuxième tableau est 
Diversity of Research in Health Journal / Revue de la Diversité de la Recherche en Santé Vol 4, January / Janvier 2021 - ISSN: 2561 -1666 DOI : 10.28984/drhj.v4i1.327

moins performant, mais peut être favorable lors de l'analyse de textes plus longs, par exemple, des romans. Par contre, le premier tableau est plus performant puisque la longueur du texte est souvent en lien avec la complexité d'un texte : plus le texte est long, plus il a tendance à augmenter en complexité. Alors, aux fins de cette étude, le premier tableau qui considère la longueur du texte a été utilisé. Ce tableau a retenu 14 variables telles qu'énumérées précédemment afin d'attribuer un niveau de complexité aux livres.

Les données suivantes ont été priorisées dans cette étude : le niveau de lecture selon Fountas et Pinnell (2012) (A à O), le niveau scolaire selon Escalire (cf. tableau de corrélation https://www.cheneliere.ca/FTP/livre_numerique/Tableau_de_correlation.pdf), les variables fournies par SATO et la valeur de l'indice (aussi fournie par SATO). Celles-ci ont été placées dans un chiffrier MS Excel (Microsoft Corporation, 2018) afin de comparer les données de chaque texte et de bien préparer la matrice Stastical Package for the Social Sciences (SPSS) (IBM, 2019) version 26 pour procéder à l'analyse des données. Lorsque ce document a été créé, les chercheuses ont pu remarquer des endroits où des ajustements étaient nécessaires. C'est-à-dire que les valeurs qui semblaient démesurées par rapport à l'indice attribué ont été étudiées de plus près, comme c'est le cas des valeurs qui semblaient plus élevées que le niveau attribué au livre, même à la suite de l'élimination des onomatopées et des interjections. Lorsque les chercheuses ne pouvaient pas expliquer la raison d'être de la valeur démesurée par ce qui serait attendu en théorie, une révision approfondie de ces textes a été faite afin de tenter d'expliquer ou de justifier les valeurs trop élevées ou trop faibles. Dans certains cas, la révision des textes a permis de comprendre et de justifier la validité de l'indice (par exemple, un texte ayant un pourcentage d'adjectifs très élevé qui avait, en effet un grand nombre d'adjectifs). Dans un tel cas, la variable a été conservée. Par contre, dans d'autres cas, les variables élevées ne contribuaient pas faire augmenter le niveau de complexité du livre (par exemple, un texte pouvait avoir un pourcentage de mots non familiers élevés, mais les mots employés étaient connus par l'élève). Dans ce cas, les mots non familiers ont été éliminés du calcul, et la moyenne recalculée pour chacun de ces livres. Rappelons qu'il y a 14 variables et 156 livres, ce qui équivaut à 2184 indicateurs. Les livres comptent 49204 mots en tout. Au total, des modifications sur seulement 165 mots ou ponctuations ont été apportées sur 156 livres et 49204 mots, ce qui équivaut à un changement sur 0,3\% des mots. Des modifications ont été apportées aux endroits suivants : la valeur du pourcentage de mots non familiers (inconnus), la valeur du pourcentage de forme fonctionnelle difficile + vous, la valeur du pourcentage de « tu », la valeur du pourcentage de «! » (point d'exclamation), la valeur du pourcentage de pronoms relatifs, la valeur du pourcentage de mots de 9 lettres ou plus, la valeur du pourcentage de «en », la valeur du pourcentage de «l' », la valeur du pourcentage de verbes conjugués, la valeur du pourcentage d'adjectifs et la valeur du pourcentage de phrases contenant plusieurs mots non familiers. Dans l'éventualité où la complexité du livre telle qu'attribuée par SATO ne s'expliquait ou ne se justifiait pas facilement par les théories qui existent, cette valeur a été recalculée : c'est-àdire que lorsque la valeur trop élevée d'un livre ne rendait pas la lecture plus complexe, par exemple, cette valeur a été modifiée (comme c'est le cas des nombreuses onomatopées que l'on trouve dans des livres d'enfants). 
Diversity of Research in Health Journal / Revue de la Diversité de la Recherche en Santé Vol 4, January / Janvier 2021 - ISSN: 2561 -1666ＤOI : 10.28984/drhj.v4i1.327

Les livres des niveaux $\mathrm{G}, \mathrm{K}$ et $\mathrm{N}$ correspondent à deux niveaux scolaires. Le niveau $\mathrm{G}$ chevauche la $1^{\text {re }}$ et la $2^{\mathrm{e}}$ année, le niveau $\mathrm{K}$ chevauche la $2^{\mathrm{e}}$ et la $3^{\mathrm{e}}$ année et le niveau $\mathrm{N}$ chevauche la $3^{\mathrm{e}}$ et la $4^{\mathrm{e}}$ année. Dans ces cas, les niveaux scolaires supérieurs ont été utilisés aux fins de comparaison afin de permettre une plus grande générosité sur les acquis. À titre d'exemple le niveau $\mathrm{K}$ a été attribué à la $3^{\mathrm{e}}$ année afin de permettre aux élèves jusqu'à la $3^{\mathrm{e}}$ année de démontrer la maîtrise de ces textes. Autrement dit, lorsque la lettre qui classifie un niveau en lecture chevauche 2 niveaux scolaires (soit $1^{\text {re }} / 2^{\mathrm{e}}$, soit $2^{\mathrm{e}} / 3^{\mathrm{e}}$, soit $3^{\mathrm{e}} / 4^{\mathrm{e}}$ année), ce regroupement de livres a été retenu comme ciblant le niveau scolaire supérieur afin de ne pas sous-estimer les habiletés des lecteurs. Le niveau $\mathrm{G}$ a alors été classé comme correspondant à la $2^{\mathrm{e}}$ année, le niveau $\mathrm{K}$ à la $3^{\mathrm{e}}$ année et le niveau $\mathrm{N}$ à la $4^{\mathrm{e}}$ année. Le tableau 2 qui figure en annexe a été employé afin de faire correspondre les niveaux scolaires aux niveaux de l'échelle de Fountas et Pinnell (2012) (Chenelière, 2014).

\section{Résultats}

Les chercheuses ont voulu savoir s'il y avait corrélation entre les niveaux de lecture attribués aux livres de la série Escalire et le niveau de complexité indiqué par le système d'analyse de texte par ordinateur (SATO) (question de recherche 1). Le coefficient de corrélation, soit le Rho de Spearman a été privilégié afin de déterminer dans quelle mesure les niveaux de classement des livres de la série Escalire correspondent au niveau de complexité telle qu'attribué par SATO. Les résultats ont indiqué qu'il existe une corrélation de taille modérée significative $(r=0,668, p<0,001)$ entre les niveaux de classement des livres d'Escalire et les niveaux de complexité ciblés par SATO.

Pour ce qui est du pourcentage d'accord entre les niveaux scolaires ciblés par les livres de la série Escalire et l'indice du système d'analyse de texte par ordinateur (SATO) recalculé, somme toute, il y avait accord sur le niveau scolaire correspondant au classement de $35,3 \%$ des livres de la série. Il est important à noter que 60,3\% des livres se trouvent à un niveau près (soit à un seul niveau plus bas, ou à un niveau plus élevé) de ce qui a été recommandé par SATO. En tenant compte de ces données plus inclusives, 95,6\% des livres se trouvent soit au même niveau d'après les deux échelles ou à un niveau près.

Les chercheuses ont voulu savoir s'il existe une corrélation entre les niveaux de complexité en lecture de la série Escalire selon deux échelles différentes : celle identifiée par l'éditeur, et celle identifiée par SATO. La question suivante a alors été posée : quelle est la corrélation entre les niveaux de lecture attribués aux livres de la série Escalire et le niveau de complexité indiqué par le système d'analyse de texte par ordinateur (SATO)?

Les résultats ont montré une corrélation modérée entre les niveaux de classement des livres d'Escalire et les niveaux de complexité tels que fournis par SATO ( $\mathrm{r}=0,668, p<0,001)$. Donc, plus un livre est jugé complexe par SATO, plus il figure parmi les niveaux scolaires plus élèves selon l'éditeur d'Escalire. Inversement, moins un livre est jugé complexe par SATO, plus il a tendance à figurer parmi les niveaux de classement inférieurs. 
Diversity of Research in Health Journal / Revue de la Diversité de la Recherche en Santé Vol 4, January / Janvier 2021 - ISSN: 2561 -1666 DOI : 10.28984/drhj.v4i1.327

Les chercheuses ont également voulu savoir si les niveaux scolaires ciblés par les livres de la série Escalire sont comparables à ceux proposés par l'indice de SATO. La question suivante a alors été posée : quel est le pourcentage d'accord entre les niveaux scolaires ciblés par les livres de la série Escalire et l'indice recalculé du système d'analyse de texte par ordinateur (SATO) ? C'està-dire, combien de livres sont classés au même niveau scolaire par les deux échelles ? Les résultats sur le pourcentage d'accord figurent dans le tableau 3.

Le pourcentage d'accord entre les livres qui ont été classés comme étant un livre de niveau de $1^{\text {re }}$ année et par SATO, et par l'éditeur de la série Escalire est de $80 \% ; 26,3 \%$ des livres ont été classés comme appartenant à la $2^{\mathrm{e}}$ année par ces deux systèmes ; 33,3\% des livres classés par SATO comme étant un niveau de $3^{\mathrm{e}}$ années ont aussi classé dans ce même niveau scolaire qu'Escalire ; 63,6\% des livres classés comme étant un niveau de $4^{\mathrm{e}}$ année selon SATO et Escalire.

Les résultats ont aussi démontré une différence de classement de livres entre Escalire et SATO. Escalire classe 67 livres en $1^{\text {re }}$ année tandis que SATO les classe en $2^{\mathrm{e}}$ année. Escalire classe 5 livres en $1^{\text {re }}$ année tandis que SATO les classe en $3^{\mathrm{e}}$ année. Escalire classe 3 livres en $2^{\mathrm{e}}$ année tandis que SATO les classe en $1^{\text {re }}$ année. Escalire classe 14 livres en $2^{\mathrm{e}}$ année tandis que SATO les classe en $3^{\mathrm{e}}$ année. Escalire classe 2 livres en $3^{\mathrm{e}}$ année tandis que SATO les classe en $2^{\mathrm{e}}$ année. Escalire classe 4 livres en $3^{\mathrm{e}}$ année tandis que SATO les classe en $4^{\mathrm{e}}$ année. Escalire classe 1 livre en $3^{\mathrm{e}}$ année tandis que $\mathrm{SATO}$ le classe en $5^{\mathrm{e}}$ année. Escalire classe 1 livre en $4^{\mathrm{e}}$ année tandis que SATO le classe en $2^{\mathrm{e}}$ année. Escalire classe 3 livres en $4^{\mathrm{e}}$ année tandis que SATO les classe en $3^{\mathrm{e}}$ année. Escalire classe 1 livre en $4^{\mathrm{e}}$ année tandis que SATO les classe en $5^{\mathrm{e}}$ année. Ces résultats sont aussi démontrés dans le tableau 3.

En se basant sur ces résultats, on peut confirmer que le classement de la série d'Escalire est plus exigeant que celui de SATO puisqu'Escalire classe plus de livres à un niveau inférieur à ce qui est proposé par SATO, ce qui signifie que selon Escalire, les élèves doivent acquérir un niveau de lecture plus complexe à un niveau scolaire quelconque que ce que SATO suggère. Si les données n'avaient pas été ajustées légèrement, alors plusieurs livres relativement simples auraient été classés comme étant plus complexes, ce qui aurait pour résultat que certains livres seraient classés à des niveaux scolaires supérieurs que ce à quoi il serait attendu. À titre d'exemple, un livre contenant plusieurs onomatopées se verrait classé en $9^{\mathrm{e}}$ année, en raison de tous les mots jugés inconnus ou non familiers par SATO alors que les élèves peuvent faire la part des choses une fois qu'ils ont été exposés à ladite onomatopée.

\section{Discussion}

Le premier objectif de cette étude était de mesurer la corrélation entre les niveaux de lecture attribués aux livres de la série Escalire et le niveau de complexité indiqué par SATO CALIBRAGE. Les chercheuses ont anticipé que les niveaux de complexité attribués par SATO ainsi que ceux attribués par Escalire suivraient la même tendance, c'est-à-dire que les livres moins complexes se trouveraient dans les niveaux scolaires inférieurs et les livres les plus complexes se trouveraient dans les niveaux scolaires plus avancés. La corrélation significative et modérément forte indique alors que les niveaux de complexité des livres attribués par SATO suivent 
Diversity of Research in Health Journal / Revue de la Diversité de la Recherche en Santé Vol 4, January / Janvier 2021 - ISSN: 2561 -1666ＤOI : 10.28984/drhj.v4i1.327

passablement la même hiérarchie de complexité des livres que ce qui a été assigné par Escalire. Par contre, il ne faut pas négliger que la corrélation n'est pas parfaite. Le coefficient de Spearman indique une absence de corrélation sur plusieurs livres, indiquant un manque d'accord entre SATO et Escalire pour ce qui est du classement des livres par niveaux scolaires : certains livres sont classés soit dans un niveau trop inférieur, soit dans un niveau trop élevé.

La question qui s'ensuivait se voulait de déterminer le pourcentage d'accord entre les niveaux scolaires ciblés par ces deux échelles. Les chercheuses ont conclu qu'il y a accord sur $35,3 \%$ des livres, ce qui signifie que pour $64,7 \%$, les livres ne correspondent pas aux mêmes niveaux dans les deux échelles. Par contre, $60,3 \%$ des livres se trouvent à un niveau près (soit un niveau inférieur ou un niveau supérieur). C'est-à-dire qu'un livre classé au niveau de la $2^{\mathrm{e}}$ année à une échelle serait classé au niveau de $1^{\text {re }}$ ou de $3^{\mathrm{e}}$ année par l'autre échelle.

Une analyse supplémentaire des livres auxquels certaines variables étaient jugées comme étant démesurées a permis aux chercheuses de comprendre qu'il y a certains moments où le jugement clinique et professionnel du personnel enseignant et des experts en matière de lecture doit être privilégié à SATO-CALIBRAGE. Il faut donc utiliser un jugement critique lorsque l'on utilise ce système. À titre d'exemple, le livre «Le sirop » a été classé comme niveau de $1^{\text {re }}$ année par Escalire tandis que SATO lui a attribué un niveau de $2^{\mathrm{e}}$ année. Selon SATO, ce livre avait 2 variables très élevées : le pourcentage de mots non familiers et le pourcentage de mots de 9 lettres ou plus. Après avoir fait une analyse plus détaillée des mots contenus dans ce livre, les chercheuses ont pu conclure qu'il ne semblait pas y avoir de mots, à leur connaissance, qui ne seraient pas compris par les élèves en $1^{\text {re }}$ année. Par exemple, le mot «attention » est répété plusieurs fois dans ce livre. Bien que ce mot comprenne 9 lettres, les chercheuses ont conclu qu'il est facilement compris par les élèves en raison de sa fréquence d'occurrence. Alors, pour ce livre, elles ont donné raison à l'éditeur quant au classement du livre par niveau scolaire. Le livre «Un pirate à la mer », quant à lui, a été classé comme niveau de $2^{\mathrm{e}}$ année d'après l'échelle employée par SATO tandis qu'Escalire lui a attribué un niveau de complexité inférieur, soit de $1^{\text {re }}$ année. SATO avait accordé à ce livre un pourcentage d'adjectifs élevés. Une analyse plus approfondie des adjectifs compris dans ce livre a pu confirmer que ce texte contenait un grand nombre d'adjectifs, tels que « colorés », « magnifique » et « souriants ». L'analyse de la fréquence des adjectifs employés, et des mots non familiers permettent de confirmer que le niveau attribué par SATO est plus fiable que celui d'Escalire.

Somme toute, l'accord sur le classement des livres entre les deux systèmes est passable. En tenant compte des particularités de certains indicateurs, le personnel enseignant est en mesure d'estimer à quel moment les livres devraient être présentés à l'élève et dans quelle mesure l'élève d'un certain niveau scolaire devrait pouvoir lire les livres avec aisance. Comme toute autre intervention, il en revient au personnel enseignant à adopter une philosophie d'enseignement qui favorise la différenciation pédagogique : c'est-à-dire que les livres devraient être choisis soigneusement pour rejoindre l'élève là où il ou elle est dans ses apprentissages. 
Diversity of Research in Health Journal / Revue de la Diversité de la Recherche en Santé

Vol 4, January / Janvier 2021 - ISSN: 2561 -1666 DOI : 10.28984/drhj.v4i1.327

\section{Limites de la recherche}

Cette étude est limitée par le fait que l'interprétation qui peut être faite des variables telles qu'attribuées par SATO nécessite tout de même une réflexion plus critique, plus approfondie. Dans le cas de SATO, il s'agit d'un ordinateur qui effectue des calculs. Bien que ces calculs soient un moyen objectif de mesurer les éléments d'un texte, des mots étant jugés comme non familiers sont connus par les élèves, comme plusieurs mots à plus de 9 lettres faisant partie de mots fréquemment lus par les élèves (p.ex., attention, aujourd'hui, finalement, important (Ministère de l'Éducation, des Loisirs et des Sports, 2014²). C'est la raison pour laquelle une analyse plus profonde en exerçant un jugement critique fondé sur une analyse lexicale et linguistique adaptée à la région dans laquelle évolue l'élève était requise afin de mieux interpréter les données qui ont été produites.

La recherche a été quelque peu limitée par le fait que l'interprétation des données aurait été plus juste s'il avait été facile à repérer les raisons théoriques d'employer les indicateurs qu'utilise SATO. Ces fondements théoriques n'ont pas pu être repérés dans la documentation. Qui plus est, il n'est pas clair pour les chercheuses en lesquels l'utilisation du « l'» ou des points d'exclamation, par exemple, fournissent un indice de complexité sur le texte. Sans vouloir contester le fait que ces indicateurs aient été retenus, il devient difficile pour les chercheuses d'appuyer les résultats sans ces fondements théoriques. Un travail de validation comme ce qu'a voulu être cette étude ne peut pas se faire à l'extérieur d'un exercice de comparaison soit à un autre outil, soit à partir d'un échantillonnage sur le terrain auprès d'élèves. Cette étude a privilégié la comparaison entre l'échelle SATO et les livres Escalire classés selon l'échelle Fountas et Pinnell (2012) comme SATO se veut être une mesure qui permet de classer les livres en niveaux de complexité, mais une piste future consistera à faire valider la série Escalire en Ontario par l'entremise d'exercice de lecture auprès d'élèves de la $1^{\text {re }}$ à la $4^{\mathrm{e}}$ année.

\section{Pistes futures}

Cette recherche a soulevé un questionnement quant à l'absence de validation auprès d'élèves franco-ontariens par l'entremise de la série Escalire. Pour une étude future, il serait intéressant de faire lire les livres de la série Escalire par des élèves des niveaux ciblés, afin de savoir si les niveaux de classement auxquels les livres ont été attribués sont effectivement lus correctement et bien compris par les élèves dudit niveau scolaire ciblé. Une telle étude permettrait de confirmer ou d'infirmer ce qui est avancé au sujet du choix de mots (familiers/non familiers) et d'adjectifs dans les textes.

\section{Conclusion}

Les résultats de cette recherche indiquent qu'il existe une corrélation significative et modérément forte entre les niveaux de lecture attribués aux livres de la série Escalire par l'éditeur et le niveau de complexité comme indiqué par SATO. Par contre, les résultats indiquent aussi que

\footnotetext{
${ }^{2}$ Comme SATO-CALIBRAGE (Daoust et coll., 1996) et la série Escalire (2014) ont été créés ou publiés au Québec, et compte tenu du manque de listes de mots fréquents en lecture publiées en Ontario, la recherche sur les mots fréquents contenant plus de 9 lettres a été faite parmi les listes disponibles et accessibles auprès du MELS (2014).
} 
Diversity of Research in Health Journal / Revue de la Diversité de la Recherche en Santé

Vol 4, January / Janvier 2021 - ISSN: 2561 -1666 DOI : 10.28984/drhj.v4i1.327

les deux systèmes (SATO et Escalire) ont parfois besoin d'interprétation humaine pour mieux comprendre où classer les livres. Puisque SATO fournit des variables purement objectives, il est important d'exercer un jugement critique pour passer à l'analyse de l'information. Il est donc nécessaire de tenir compte des variables qui sont de nature plus qualitative afin de justifier les niveaux de complexité déjà attribués aux livres de la série Escalire. De plus, les résultats démontrent que le pourcentage d'accord entre les deux échelles est de 35,3\%. À première vue, ce pourcentage peut sembler assez faible. Toutefois, lorsque sont considérés les livres qui sont au même niveau indépendamment de l'échelle (SATO ou Escalire) en plus de ceux se trouvant à un niveau près de l'échelle comparative (soit un niveau inférieur ou un niveau supérieur), alors le pourcentage d'accord augmente notablement à 95,6\%. Ceci indique que les deux échelles, incluant les quelques modifications apportées par les chercheuses, ne sont pas aussi distantes qu'elles le laissaient entendre après la première analyse. Les deux échelles ont tout de même classé les livres soit dans le même niveau scolaire, ou très près du niveau scolaire attribué par l'autre échelle. Les mesures de lisibilité des textes qui étaient hautement intuitives il y a une génération (Fulcher, 1997) le sont beaucoup moins à l'heure actuelle en raison des avancées technologiques (Benjamin, 2012) qui permettent ce genre d'étude d'être menée à terme.

\section{Remerciements}

Les chercheuses aimeraient reconnaître la contribution de Santé Canada dans cette étude sans laquelle elle n'aurait pas été menée à bonne fin. 
Diversity of Research in Health Journal / Revue de la Diversité de la Recherche en Santé

Vol 4, January / Janvier 2021 - ISSN: 2561 -1666 DOI : 10.28984/drhj.v4i1.327

\section{Références}

Benjamin, R.G. (2012). Reconstructing Readability: Recent Developments and Recommendations in the Analysis of Text Difficulty. Educational Psychological Review, 24, 63-88. doi : $10.1007 / \mathrm{s} 10648-011-9181-8$

Chenelière Éducation (2014). Tableau de corrélation. Collection en littératie et en numératie. https://www.cheneliere.ca/FTP/livre_numerique/Tableau_de_correlation.pdf

Cunningham, J.W., Spadorcia, S.A., Erickson, K.A., Koppenhaver, D.A., Strum, J.M. et Yoder, D.E. (2005). Investigating the instructional supportiveness of leveled texts. Reading Research Quarterly, 40(4), 410-427. doi : 10.1598/RRQ.40.4.2

Daoust, F. Laroche, L. et Ouellet, L., (1996). SATO-CALIBRAGE : présentation d'un outil d'assistance au choix et à la rédaction de textes pour l'enseignement. Revue québécoise de linguistique, 5(1), 205-234. doi : 10.7202/603132

Éditions Passe-Temps. (2014). Escalire. Les Éditions Passe-Temps. Repéré à http://www.escalire.com/index.html

Fountas, I. C. et Pinnell, G. S. (2012). Guided reading: The romance and the reality. The Reading Teacher, 66(4), 268-284.

Fountas, I. C. et Pinnell, G. S. (1999). Matching Books to Readers: Using Leveled Books in Graded Reading, K-3. Heinemann.

Fulcher, G. (1997). Text difficulty and accessibility: Reading formulae and expert judgement. System, 25(4), 497-513. doi : 10.1016/S0346-251X(97)00048-1

Galloway-Bell, S. (2003). A Review of the Literature: The Effectiveness of Leveled Reading Groups in Improving Oral Proficiency and Comprehension to First Grade Students. (Dissertation) Educational Resource Information Center ED479119. Division of Education. School of Business, Education and Leadership, Dominican University of California.

Guthrie, J. T. et Davis, M. H. (2003). Motivating struggling readers in middle school through an engagement model of classroom practice, Reading \& Writing Quarterly, 19(1), 5985, doi : 10.1080/10573560308203

Hastings, K. (2016). Leveled Reading and Engagement with Complex Texts. Reading Improvement, 53(2), 65-71. EJ1104262

Léonard, B. (2012). Lecture des mots fréquents. Brigitte Prof. http://brigitteprof.brigitteleonard.com/2012/03/18/lecture-des-mots-frequents/

Ministère de l'éducation de 1'Ontario (2003). Stratégie de lecture au primaire de 1'Ontario. Guide d'enseignement efficace de la lecture, de la maternelle à la $3 e$ année. http://www.atelier.on.ca/edu/resources/guides/GEE_Lecture_M_3.pdf

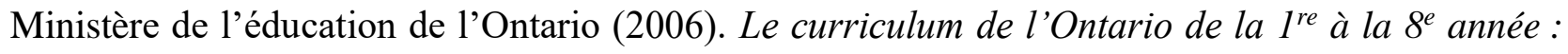
Français - Révisé. Toronto, chez l'auteur.

Ministère de l'Éducation, des Loisirs et des Sports (2006). Histoire et Éducation à la Citoyenneté. Document de travail Programme de formation de l'école québécoise, secondaire, 2e cycle, Québec, Ministère de l'Éducation. 
Diversity of Research in Health Journal / Revue de la Diversité de la Recherche en Santé

Vol 4, January / Janvier 2021 - ISSN: 2561 -1666 DOI : 10.28984/drhj.v4i1.327

Ministère de l'Éducation, des Loisirs et des Sports (2014). Programme de formation de l'école Québécoise : enseignement primaire. Liste orthographique à l'usage des enseignantes et des enseignants, Québec, Ministère de l'éducation,

http://www.education.gouv.qc.ca/fileadmin/site_web/documents/dpse/formation_jeunes/List eOrthographique_Primaire.pdf

Pitcher, B et Fang, Z. (2007). Can we trust levelled texts? An examination of their reliability and quality from a linguistic perspective. Literacy, 41(1), 43-51. doi: 10.1111/j.14679345.2007.00454.x

Seals, M. P. (2013). Impact of Leveled Reading Books on the Fluency and

Comprehension Levels of First Grade Students. (Doctoral dissertation) Educational Resource Information Center ED564864. Liberty University.

Tiernan, B. (2016). The Role of Levelled Readers in Supporting the Development of Literacy. Journal of the Irish Learning Support Association, 38, 25-33.

Vygotsky, L. (1978). Interaction Between Learning and Development. Dans Gauvain et Cole (dir.), Readings on the development of children (2e éd) (pp. 29-36). New York: W. H. Freeman and Company. 
Diversity of Research in Health Journal / Revue de la Diversité de la Recherche en Santé Vol 4, January / Janvier 2021 - ISSN: 2561 -1666 DOI : 10.28984/drhj.v4i1.327

\section{Annexe}

Tableau 2: Les niveaux scolaires suggérés par rapport aux niveaux de l'échelle de Fountas et Pinnell (2012) et (Chenelière 2014)

\begin{tabular}{ll}
\hline Niveaux de l'échelle de & Niveaux scolaires suggérés, \\
Fountas et Pinnell (2012) & français langue maternelle \\
\hline A & $1^{\text {re }}$ \\
B & $1^{\text {re }}$ \\
C & $1^{\text {re }}$ \\
D & $1^{\text {re }}$ \\
E & $1^{\text {re }}$ \\
F & $1^{\text {re }}$ \\
G & $1^{\text {re }-2^{\mathrm{e}}}$ \\
H & $2^{\mathrm{e}}$ \\
I & $2^{\mathrm{e}}$ \\
J & $2^{\mathrm{e}}$ \\
K & $2^{\mathrm{e}}-3^{\mathrm{e}}$ \\
L & $3^{\mathrm{e}}$ \\
M & $3^{\mathrm{e}}$ \\
N & $3^{\mathrm{e}}-4^{\mathrm{e}}$ \\
O & $4^{\mathrm{e}}$ \\
\hline
\end{tabular}


Diversity of Research in Health Journal / Revue de la Diversité de la Recherche en Santé Vol 4, January / Janvier 2021 - ISSN: 2561 -1666 DOI : 10.28984/drhj.v4i1.327

Tableau 3 : Corrélation entre les niveaux scolaires selon Escalire et les niveaux scolaires attribués par SATO

Escalire Système d'analyse d'un texte par ordinateur (SATO)

\begin{tabular}{|c|c|c|c|c|c|}
\hline & $1^{\mathrm{re}}$ année & $2^{\mathrm{e}}$ année & $3^{\mathrm{e}}$ année & $4^{\mathrm{e}}$ année & $5^{\mathrm{e}}$ année \\
\hline \multirow[t]{2}{*}{$1^{\text {re }}$ année } & 12 & 67 & 5 & 0 & 0 \\
\hline & $80 \%$ & $70,5 \%$ & $15,2 \%$ & & \\
\hline \multirow[t]{2}{*}{$2^{\mathrm{e}}$ année } & 3 & 25 & 14 & 0 & 0 \\
\hline & $20 \%$ & $26,3 \%$ & $42,4 \%$ & & \\
\hline \multirow[t]{2}{*}{$3^{\mathrm{e}}$ année } & 0 & 2 & 11 & 4 & 1 \\
\hline & & $2,1 \%$ & $33,3 \%$ & $36,4 \%$ & $50 \%$ \\
\hline \multirow[t]{2}{*}{$4^{\mathrm{e}}$ année } & 0 & 1 & 3 & 7 & 1 \\
\hline & & $1,1 \%$ & $9,1 \%$ & $63,6 \%$ & $50 \%$ \\
\hline Total & 15 & 95 & 33 & 11 & 2 \\
\hline
\end{tabular}

Cet article a été expertisé par :

1. Chantal Mayer-Crittenden, Ph.D., professeure agrégée.

École d'Orthophonie, Université Laurentienne.

2. Mélanie Perron, Ph.D., professeure agrégée.

Département de psychologie, Université Laurentienne.

3. Athanase Simbagoye, Ph.D., professeur titulaire

École d'éducation, Université Laurentienne. 